OPEN ACCESS

Edited by:

Tianmu Chen,

Xiamen University, China

Reviewed by:

Meghnath Dhimal,

Nepal Health Research Council, Nepal

Tao Liu,

Guangdong Provincial Center for Disease Control and Prevention, China

*Correspondence: Zhicong Yang zhicongyanggz@163.com

tThese authors have contributed equally to this work and share first authorship

Specialty section

This article was submitted to Infectious Diseases - Surveillance, Prevention and Treatment, a section of the journal Frontiers in Medicine

Received: 13 February 2021 Accepted: 31 May 2021 Published: 28 July 2021

Citation:

Lu J, Liu Y, Ma X, Li M and Yang Z (2021) Impact of Meteorological Factors and Southern Oscillation Index on Scrub Typhus Incidence in Guangzhou, Southern China, 2006-2018. Front. Med. 8:667549

doi: 10.3389/fmed.2021.667549

\section{Impact of Meteorological Factors and Southern Oscillation Index on Scrub Typhus Incidence in Guangzhou, Southern China, 2006-2018}

\author{
Jianyun $\mathrm{Lu}^{1 \dagger}$, Yanhui $\mathrm{Liu}^{1 \dagger}$, Xiaowei $\mathrm{Ma}^{2 \dagger}$, Meixia $\mathrm{Li}^{1}$ and Zhicong Yang ${ }^{3 *}$ \\ ${ }^{1}$ Department of Infectious Disease Control and Prevention, Guangzhou Center for Disease Control and Prevention, \\ Guangzhou, China, ${ }^{2}$ Department of Public Health Emergency Preparedness and Response, Guangzhou Center for Disease \\ Control and Prevention, Guangzhou, China, ${ }^{3}$ Guangzhou Center for Disease Control and Prevention, Guangzhou, China
}

Background: Scrub typhus was epidemic in the western Pacific Ocean area and East Asia, scrub typhus epidemic in densely populated areas in southern China. To better understand the association between meteorological variables, Southern Oscillation Index (SOI), and scrub typhus incidence in Guangzhou was benefit to the control and prevention.

Methodology/Principal Findings: We collected weekly data for scrub typhus cases and meteorological variables in Guangzhou, and Southern Oscillation Index from 2006 to 2018, and used the distributed lag non-linear models to evaluate the relationships between meteorological variables, SOI and scrub typhus. The median value of each variable was set as the reference. The high-risk occupations were farmer (51.10\%), house worker (17.51\%), and retiree (6.29\%). The non-linear relationships were observed with different lag weeks. For example, when the mean temperature was $27.7^{\circ} \mathrm{C}$ with1-week lag, the relative risk (RR) was highest as 1.08 (95\% Cl: 1.01-1.17). The risk was the highest when the relative humidity was $92.0 \%$ with 9-week lag, with the RR of 1.10 (95\% Cl: 1.02-1.19). For aggregate rainfall, the highest RR was 1.06 (95\% Cl: 1.03-1.11), when it was $83.0 \mathrm{~mm}$ with 4 -week lag. When the SOI was 19 with 11-week lag, the highest RR was 1.06 (95\% Cl: 1.01-1.12). Most of the extreme effects of SOI and meteorological factors on scrub typical cases were statistically significant.

Conclusion/Significance: The high-risk occupations of scrub typhus in Guangzhou were farmer, house worker, and retiree. Meteorological factors and SOI played an important role in scrub typhus occurrence in Guangzhou. Non-linear relationships were observed in almost all the variables in our study. Approximately, mean temperature, and relative humidity positively correlated to the incidence of scrub typhus, on the contrary to atmospheric pressure and weekly temperature range (WTR). Aggregate rainfall and wind velocity showed an inverse- $U$ curve, whereas the SOI appeared the bimodal distribution. These findings can be helpful to facilitate the development of the early warning system to prevent the scrub typhus.

Keywords: distributed lag non-linear models, meteorological factors, southern oscillation index, scrub typhus, early warning 


\section{INTRODUCTION}

Scrub typhus (tsutsugamushi disease), a rickettsiosis caused by Orientia tsutsugamushi, is epidemic in the western Pacific Ocean area and East Asia $(1,2)$. Recently, scrub typhus cases showed a rising trend in Asia, case-fatality rates from areas with reduced drug sensitivity were reported in South India and northern Thailand at 12.2 and $13.6 \%$, respectively (3), and it has also become an important health issue in China (4). At present, scrub typhus epidemic in densely populated areas in southern China and millions of people is at risk of the infection of scrub typhus (5). A rapid increase of scrub typhus was reported in Guangzhou in 2012, which was a nearly four-fold increase from 2006 (5). A rising trend was observed in recent years, which caused a huge disease burden in Guangzhou city. Several reasons might contribute to the increase of scrub typhus cases, such as variation of genotypes of O. tsutsugamushi (6), increased risk of exposure to vegetation with more and more parks in the city construction (7), improvement of the surveillance system, and climate change. A previous study showed that climate factors played an important role in the spread of vector-borne diseases (8).

In common, human is transmitted by the bite of infected larval trombiculid mites, with the incubation period about 4 to 21 days. Fever, eschars or ulcers, skin rash, and lymphadenopathy are typical clinical symptoms (9). Orientia-infected mites inhabit in various environments, including grassland, field, forest, and scrub. Transmission of scrub typhus rests with the seasonal activities of both chiggers and humans (10). Hence, scrub typhus mainly occurred in farmers and urban populations who have an exposure in parks and the countryside (11).

For the past few years, the effects of meteorological variables have been broadly studied as early-warning signals of underlying epidemics on some rodent-borne infectious diseases, such as, hemorrhagic fever with renal syndrome (12), lime-disease and tick-borne encephalitis (13). Previous studies showed that the transmission of scrub typhus influenced by climate. For instance, high rainfall and temperature could increase the chigger abundance, which increased the spread of scrub typhus $(5,7)$. El Niño/Southern Oscillation (ENSO) is a systematic pattern of global climate variability (14). The Southern Oscillation Index (SOI), an indicator of ENSO activity, is defined as the normalized atmospheric pressure difference between Darwin, Australia and Tahiti, South Pacific (15). The SOI has been reported to be associated with infectious diseases, including hemorrhagic fever with renal syndrome (16), and dengue fever (17). However, limited researches reported the weekly associations between climatic variables, SOI, and scrub typhus cases. Moreover, the quantitative and lagged relationships mentioned above remain to be determined. Hence, it's urgent to detect these associations to build up forecasting and early warning for scrub typhus, especially in Guangzhou, southern China, with the nationwide highest scrub typhus incidence $(4,5)$.

\footnotetext{
Abbreviations: SOI, Southern Oscillation Index; RR, relative risk; CI, confidence interval; WTR, weekly temperature range; DLNMs, distributed lag nonlinear models.
}

In our research, we used the distributed lag non-linear models (DLNMs), which can flexibly describe relationships and explore underlying lagged and non-linear effects (18), to detect the association between SOI, meteorological variables, and scrub typhus cases. Our findings can not only offer in-depth insights into the future effect of climate change on the transmission of scrub typhus in southern China, but also in subtropical zone.

\section{MATERIALS AND METHODS}

\section{Study Region}

Guangzhou is the third largest city in China, which has a registered inhabitant over 8.97 million. Guangzhou, located in southern China, has a humid subtropical climate with hot and wet summers, and cool, dry winters, which locates at $112^{\circ} 57^{\prime} \mathrm{E}$ to $114^{\circ} 3^{\prime} \mathrm{E}$ and $22^{\circ} 26^{\prime} \mathrm{N}$ to $23^{\circ} 56^{\prime} \mathrm{N}$.

\section{Data of Scrub Typhus Cases}

During the period from 2006 to 2018, data of scrub typhus cases in Guangzhou were obtained from the National Notifiable Disease Report System, which covers all community health centers and hospitals in Guangzhou. The clinical diagnosis cases should meet the criteria as follows. (1) Fever with lymphadenopathy or skin rash, (2) typical eschars or ulcers, and (3) A history of field exposure 1-3 weeks before the onset of symptoms. The laboratory confirmed cases must meet the criteria mentioned above and fulfill one of the following criteria: fourfold or more increase in serum IgG antibody titers between acute and convalescent sera-detected by indirect immune fluorescence antibody assay (IFA); The O. tsutsugamushi was detected in clinical specimens using polymerase chain reaction (PCR); the isolation of O. tsutsugamushi (19). The cases in our study contained clinical cases and laboratory confirmed cases. Scrub typhus was not the national notifiable disease; however, it required compulsory notification for doctors in Guangzhou by the local laws.

\section{Meteorological Data}

We collected the weekly meteorological data, such as mean temperature $\left({ }^{\circ} \mathrm{C}\right)$, aggregate rainfall $(\mathrm{mm})$, weekly temperature range $\left({ }^{\circ} \mathrm{C}\right)$ (WTR: it is defined as the gap between the highest and the lowest temperature in a week), wind velocity $(\mathrm{m} / \mathrm{s})$, relative humidity (\%), atmospheric pressure ( $\mathrm{hPa}$ ), and sunshine hours (hours), were collected from the Guangzhou Meteorological Bureau (GZMB), which measure the meteorological data at a fixed-site station located in 186 surveillance stations in different regions of all the 11 districts of Guangzhou city. SOI data were publicly accessible from the Australian Bureau of Meteorology (http://www.longpaddock.qld.gov.au/).

\section{Statistical Analysis}

Based on the descriptive analysis of scrub typhus cases, SOI and meteorological variables in Guangzhou, we deduced that their correlations were non-linear, which the DLNMs were suitable to detect. We used the variance inflation factor (VIF) to evaluate the co-linearity. If the VIF exceeds 5, it would be manifest 
multicollinearity (20). All the predictor variables studied in our study were tested for each model.

The quasi-Poisson function was incorporated with DLNMs, allowing over-dispersion in the scrub typhus cases. The model structure is as below:

$\log \left[\mathrm{E}\left(\mathrm{Y}_{\mathrm{t}}\right)\right]=\alpha+\sum N S_{\mathrm{i}}\left(\mathrm{X}_{\mathrm{i}}, \mathrm{df}_{\mathrm{i}}\right)+\delta$ Holiday $_{\mathrm{t}}+\mathrm{NS}($ Time, 14$)$ $+\Phi_{\mathrm{t}}$

Yt represents the scrub typhus cases in week $\mathrm{t} ; \alpha$ and $\delta$ are both the intercept; NS represents a natural cubic spline; Holiday is a binary variable, if week $t$ contains more than 7 days of public or summer/winter vacation, its value is " 1 "; and Time symbolizes long-term trends and seasonality; $\mathrm{df}$ means the degree of freedom; X represents meteorological factors and SOI. The incubation of the scrub was from 4 days to 20 days. $\Phi_{t}$ refers to the autoregressive terms of weekly flu counts on the logarithmic scale at lag 1 week to control for the autocorrelations occurring in cases of infectious disease (21). We alternated the lag week from 1 to 3 weeks, and finally selected the lag 1 week due to the The Akaike information criterion (AIC).

The AIC was applied to select the df per week for controlling long-term trends and seasonality, and the $\mathrm{df}$ for the meteorological variables and the SOI that yielded the best-fitting model. We selected NS $(\mathrm{df}=3)$ for the meteorological factors and the SOI. Different time lags of weeks were applied to evaluate the effects of meteorological variables on scrub typhus according to
AIC. The finally model structure for each variable was shown in the Supplementary Table 1.

For all meteorological variables, as the reference of the median value of each variable, we define the extremely high effect as the value in the $97.5 \%$ range and extremely low effect as the value in the $2.5 \%$ range. We then evaluated these extreme effects on the scrub typhus cases.

The level of significant difference was set as two-sided $P<$ 0.05 . R software version 3.4 .5 was utilized to analyze the data and create the DLNMs by the "dlnm" package.

We performed sensitivity analyses by altering the $\mathrm{df}$ (5-9) per year for time, and the $\mathrm{df}(3-5)$ for meteorological variables.

\section{RESULTS}

From January 1, 2006 to December 31, 2018, a total of 8,345 scrub typhus cases were reported in Guangzhou. $48.54 \%(4,051)$ were male cases and $51.46 \%(4,294)$ were female cases, with the male-to-female ratio of 0.94 . Most of the cases were clinical diagnosis cases, accounting for $89.87 \%(7,500)$. During the study period, the numbers of scrub typhus showed a rising trend, with the top three in 2012 (1,026), 2018 (965), 2014 (936). From 2006 to 2011, cases increased slowly, then the cases had a sharp rise in 2012 and fluctuated, after 2014, the incidence decreased slightly, however it increased again in 2018. The proportion of cases was $5.97 \%$ (under 18 years old), 2.70\% (from 18 to 24 years old), $56.88 \%$ (25 to 60 years old), and $34.45 \%$ (over 60

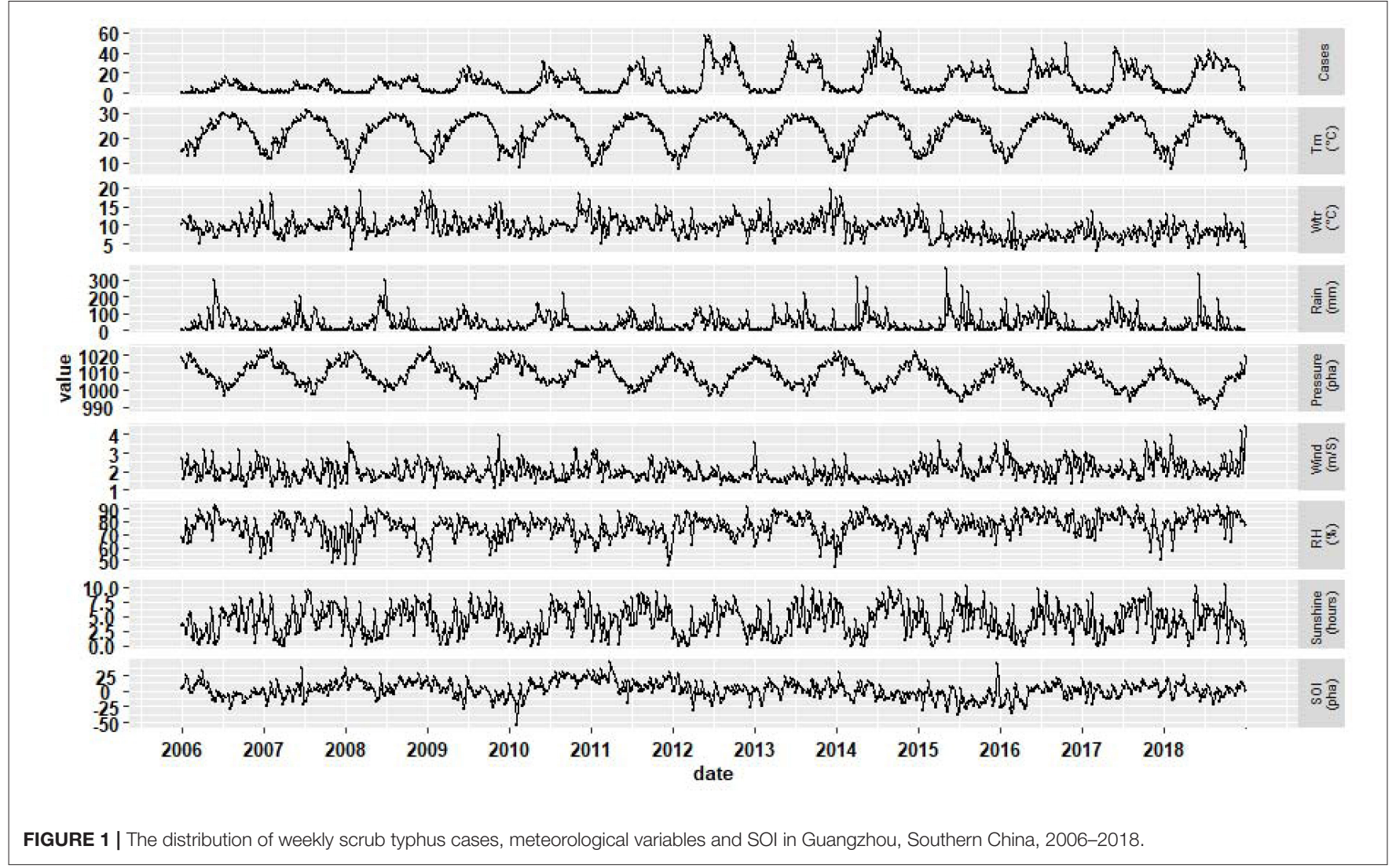


TABLE 1 | Weekly meteorological factors, SOI and scrub typhus cases in Guangzhou, 2006-2018.

\begin{tabular}{|c|c|c|c|c|c|c|c|}
\hline \multirow[t]{2}{*}{ Variables } & \multirow[t]{2}{*}{ Mean } & \multirow[t]{2}{*}{ Std. deviation } & \multicolumn{3}{|c|}{ Percentile } & \multirow[t]{2}{*}{ Min. } & \multirow[t]{2}{*}{ Max. } \\
\hline & & & 25 & 50 & 75 & & \\
\hline Cases & 12 & 12 & 2 & 6 & 19 & 0 & 59 \\
\hline Mean temperature & 22.21 & 5.92 & 17.39 & 23.45 & 27.52 & 6.15 & 31.13 \\
\hline Weekly temperature range & 9.47 & 2.75 & 7.64 & 9.21 & 10.98 & 2.97 & 19.67 \\
\hline Aggregate rainfall & 38.87 & 53.10 & 1.20 & 18.50 & 56.20 & 0.00 & 368.30 \\
\hline Atmospheric pressure & 1008.18 & 6.90 & 1003.03 & 1008.07 & 1013.63 & 988.81 & 1024.56 \\
\hline Wind velocity & 2.02 & 0.51 & 1.65 & 1.94 & 2.30 & 1.11 & 4.45 \\
\hline Relative humidity & 76.37 & 8.64 & 71.32 & 77.57 & 82.46 & 44.79 & 92.93 \\
\hline Sunshine hours & 4.40 & 2.54 & 2.33 & 4.31 & 6.38 & 0 & 10.57 \\
\hline SOI & 1.67 & 13.49 & -6.01 & 1.85 & 10.65 & -54.04 & 44.99 \\
\hline
\end{tabular}

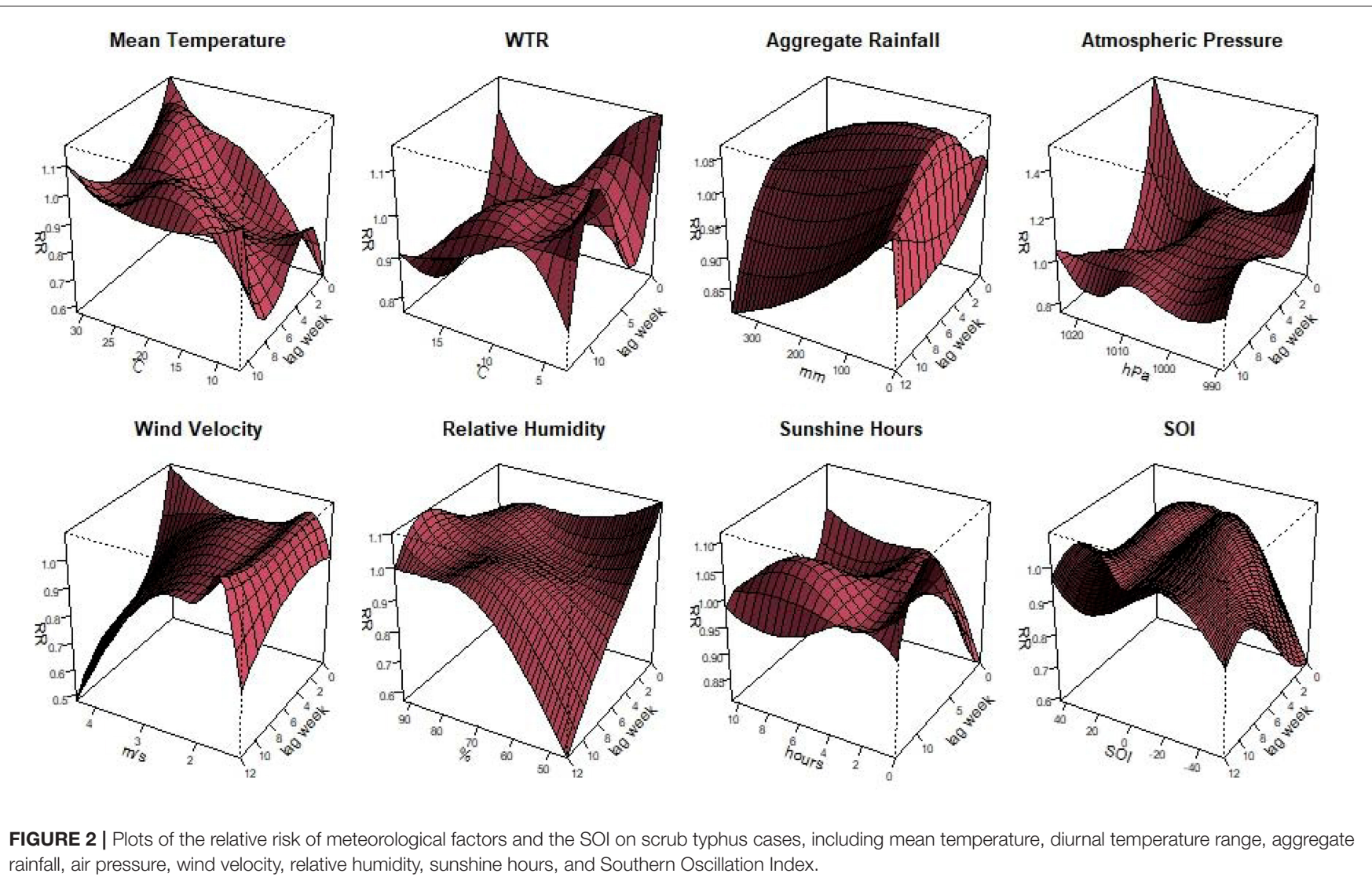

years old), respectively. The high-risk occupations were farmer (51.10\%), house worker $(17.51 \%)$, and retiree $(6.29 \%)$. During 2006-2018, 84.76\% (7,073) cases occurred from May to October, with the peak at June. (Figure 1) during the study period, Table 1 showed the weekly distribution of meteorological variables, SOI and scrub typhus cases.

The Figure 2 illustrated the non-linear relationship between the meteorological variables, SOI and scrub typhus cases in Guangzhou with different lag weeks. Different characteristics were observed in the different variables. When the mean temperature was $27.7^{\circ} \mathrm{C}$ with1-week lag, the relative risk (RR) was highest as 1.08 (95\% CI: 1.01-1.17). The risk was the highest when the relative humidity was $92.0 \%$ with 9 -week lag, with the RR of 1.10 (95\% CI: $1.02-1.19$ ). When the WTR was $6.0^{\circ} \mathrm{C}$ with 0 -week lag, the $\mathrm{RR}$ reached the high value as $1.10(95 \%$ CI: 1.18-1.20). When the wind speed was $1.6 \mathrm{~m} / \mathrm{s}$ with 2 -week lag, the highest RR was 1.04 (95\% CI: 1.00-1.08). For aggregate rainfall, the highest RR was 1.06 (95\% CI: 1.03-1.11), when it was $83.0 \mathrm{~mm}$ with 4 -week lag. As the atmospheric pressure was $989.0 \mathrm{hPa}$ with 0 -week lag, the RR reached the peak of 1.29 (95\% 


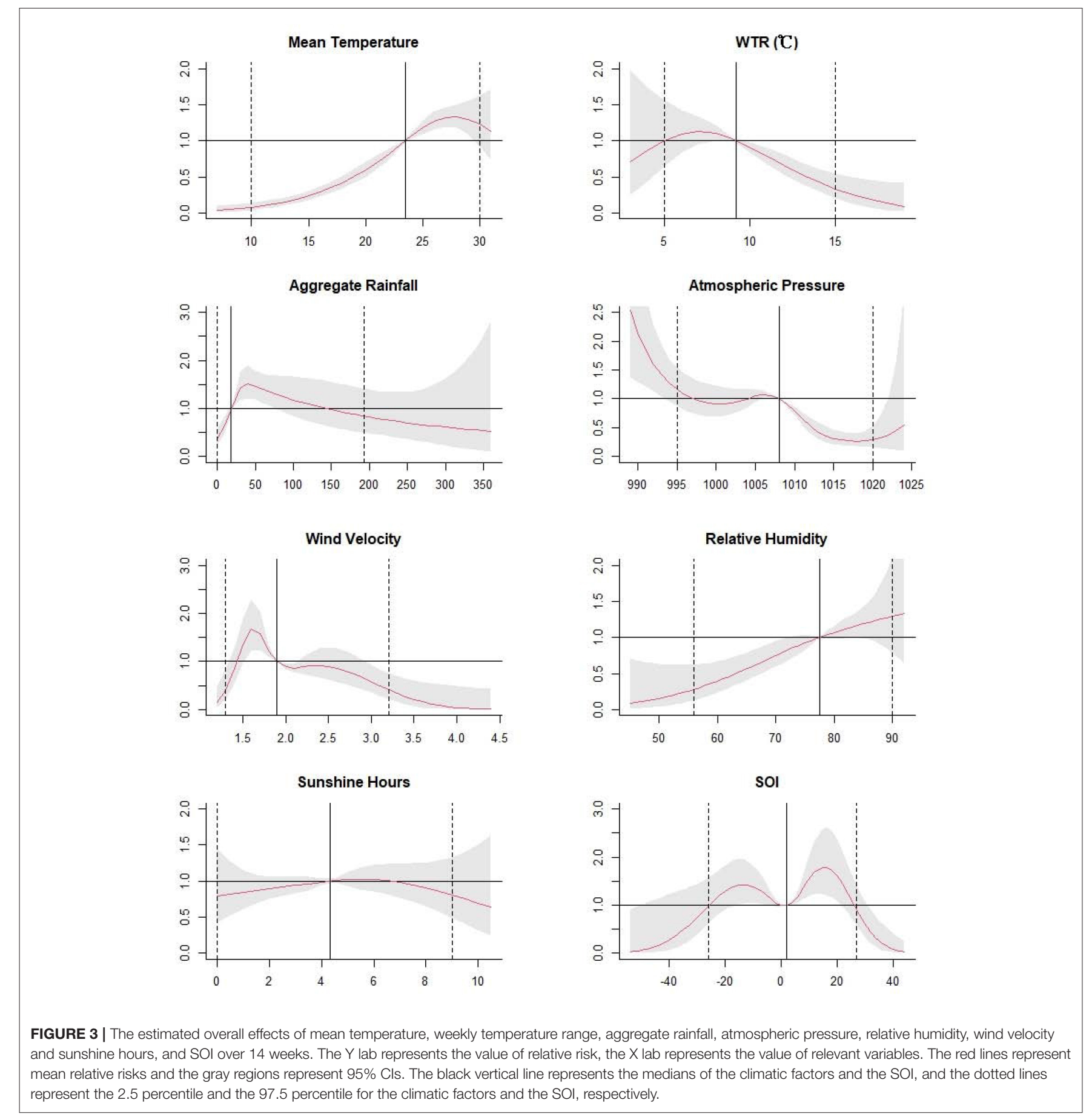

CI: 1.04-1.61). For the duration of sunshine, the RR was 1.21 (95\% CI: 1.05-1.18) when the sunshine was $0 \mathrm{~h}$ with 10 -week lag. Regarding the SOI, the highest RR was 1.06 (95\% CI: 1.01-1.12). When the SOI was 18.8 with 11-week lag.

The Figure 3 illustrated unidimensional summaries of the overall effects of meteorological factors and SOI on scrub typhus with the corresponding lag weeks. Non-linear relationships were observed. Briefly, for mean temperature, the RR and 95\% CI values showed a rising trend, which were all over 1 from $23.5^{\circ} \mathrm{C}$ to $29.6^{\circ} \mathrm{C}$. For WTR, the RR and $95 \%$ CI values revealed a decline trend, and it were over 1 from $8.1^{\circ} \mathrm{C}$ to $9.3^{\circ} \mathrm{C}$ and was $<1$ beyond $9.3^{\circ} \mathrm{C}$. For aggregate rainfall, it was statistically significant from 0 to $70 \mathrm{~mm}$, and the RR and 95\% CI values were over 1 from 19 to $76 \mathrm{~mm}$, with a peak at $38 \mathrm{~mm}$. The RR values of atmospheric pressure were downtrend, with a peak at $989 \mathrm{hPa}$. For wind velocity, the RR values were uptrend and peaked at $1.6 \mathrm{~m} / \mathrm{s}$, then it went downtrend. For relative humidity, the RR values were uptrend and statistically significant from 45 to $72 \%$. For 

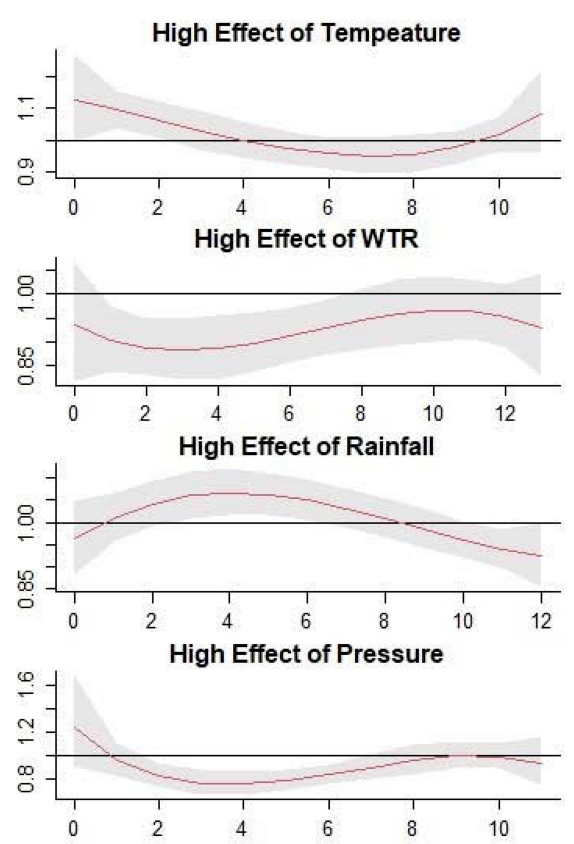

High Effect of Wind

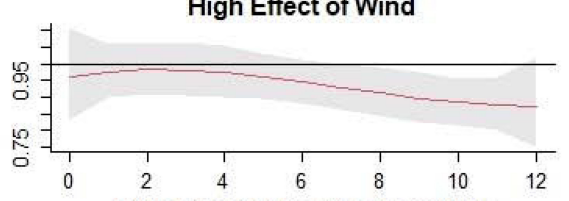

High Effect of Relative Humidity
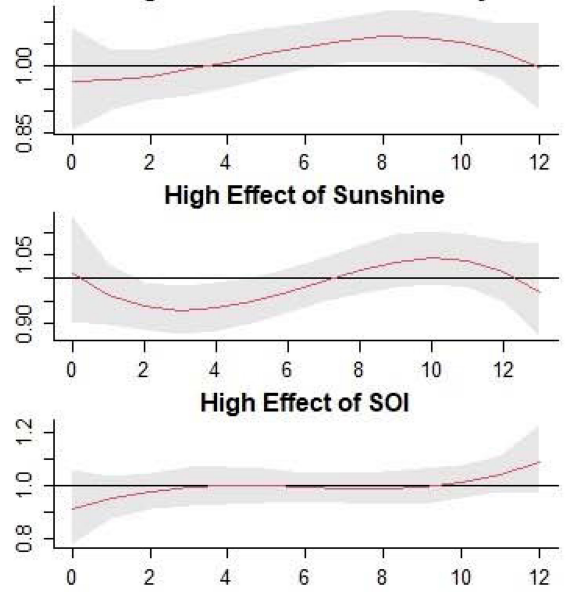

Cold Effect of Tempeature
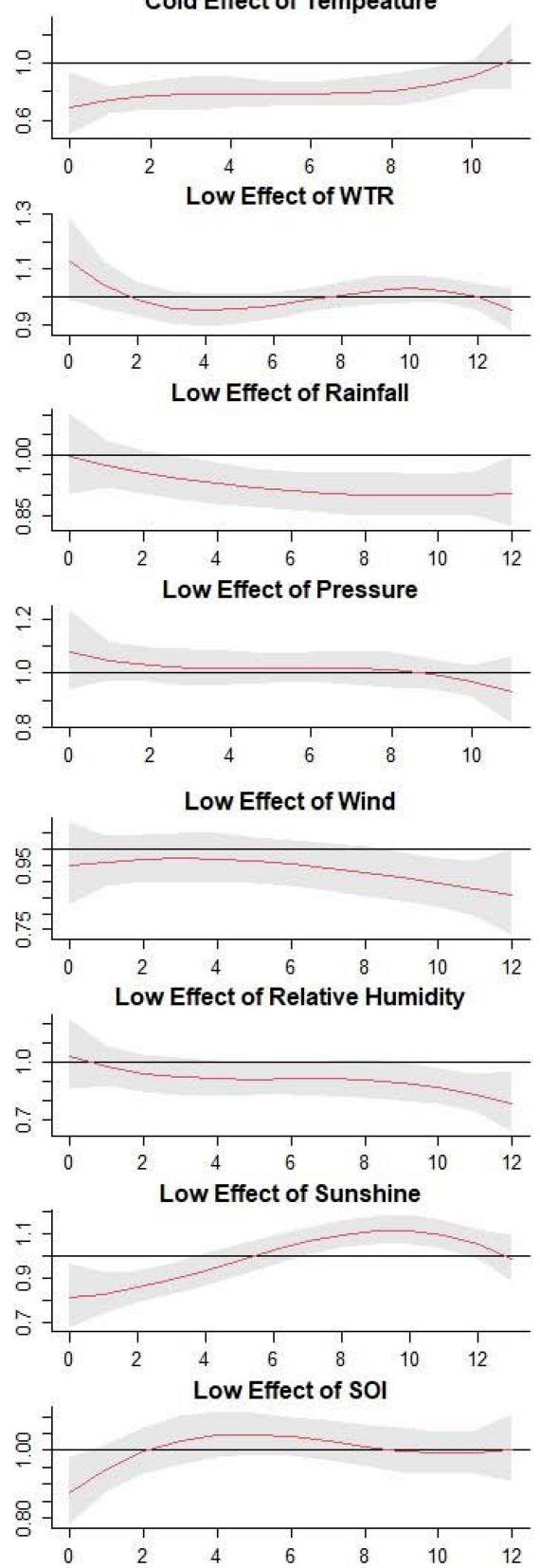

FIGURE 4 | The estimated extreme effects of mean temperature, weekly temperature range, aggregate rainfall, atmospheric pressure, wind velocity, relative humidity, hours of sunshine, and SOI with extremely high effects (97.5\%) and extremely low effects (2.5\%). The $\mathrm{Y}$ lab represents the value of relative risk, the $\mathrm{X}$ lab represents the value of lag weeks. The red lines represent mean relative risks and gray regions represent $95 \% \mathrm{Cls}$.

SOI, the RR values showed an M-shape bimodal distribution, with a peak at 15.4. The statistically significant increase of RR value was observed in two stages from -16.2 to -4.4 , and from 2.4 to 21.6 . The RR and 95CI values were lower than 1 beyond 31.0. Finally, sunshine hours did not have a statistical correlation with scrub.
The Figure 4 illustrated the extreme effects of meteorological factors and SOI on scrub typhus cases along the various lag weeks (Figure 4 and Table 2).

The sensitivity analyses revealed that similar results were generated by changing the $\mathrm{df}$ for seasonality and long-term trends and meteorological factors in our study. 
TABLE 2 | The extreme effects of meteorological variables and SOI on scrub typhus along the lag weeks in Guangzhou, Southern China, $2006-2018$.

\begin{tabular}{|c|c|c|c|c|c|c|}
\hline \multirow[t]{2}{*}{ Meteorological variables } & \multirow[t]{2}{*}{ Lag week } & \multicolumn{2}{|c|}{ High effect $(97.5 \%)$} & \multirow[t]{2}{*}{ Lag week } & \multicolumn{2}{|c|}{ Low effect $(2.5 \%)$} \\
\hline & & $\mathbf{R R}$ & $95 \% \mathrm{Cl}$ & & $\mathbf{R R}$ & $95 \% \mathrm{Cl}$ \\
\hline Mean temperature & 1 & 1.10 & $1.04-1.16$ & 0 & 0.69 & $0.50-0.94$ \\
\hline Weekly temperature range & 3 & 0.88 & $0.82-0.95$ & / & / & / \\
\hline Aggregate rainfall & 12 & 0.88 & $0.79-0.98$ & 10 & 0.90 & $0.85-0.95$ \\
\hline Atmospheric pressure & 4 & 0.76 & $0.66-0.86$ & / & / & / \\
\hline Wind velocity & 11 & 0.88 & $0.80-0.96$ & 12 & 0.86 & $0.73-0.99$ \\
\hline Relatively humidity & 8 & 1.07 & $1.01-1.12$ & 12 & 0.78 & $0.64-0.95$ \\
\hline Sunshine hours & 3 & 0.93 & $0.88-0.98$ & 0 & 0.81 & $0.67-0.97$ \\
\hline Sunshine hours & / & / & / & 10 & 1.12 & $1.05-1.19$ \\
\hline $\mathrm{SOI}$ & / & / & / & 0 & 0.88 & $0.78-0.98$ \\
\hline
\end{tabular}

The median value of each variable was set as reference.

\section{DISCUSSION}

During the study period, we found that scrub typhus cases in Guangzhou showed a sharply rising trend, consistent with previous studies in China. Scrub typhus incidence increased stably before 2012, and then after 2012, it increased rapidly year by year $(4,7)$. However, the distribution of scrub typhus cases in Guangzhou was different, where the peak of cases occurred in 2012 and fluctuated at a stable level, although the numbers of cases increased again in 2018. Scrub typhus was a local notifiable disease by law in Guangzhou, this was a possible reason for the stability of occurrence. It demonstrated that the surveillance is beneficial to prevent and control the scrub typhus.

The high-risk occupations of scrub typhus in Guangzhou were farmer, house worker, and retiree. In Guangzhou, the retiree and house worker people would often visit the park and part of them would sit on the grass. The plausible explanation was that agricultural activities and park visiting would facilitate the exposure to pathogen-carrying chigger mites $(19,22)$. However, our speculation should be explored by the detailed epidemiology investigation to the scrub typhus cases in the future.

In our study, we found a seasonal fluctuation of scrub typhus cases from May to October which was not unexpected when compared to previous studies in the southern China $(4,7)$. However, it was not consistent with the study in Japan which was biphasic with the main peak in November and December and a smaller peak in May and June. This discrepancy was mainly caused by the different geography and climate.

Meteorological variables, including temperature, rainfall, and relative humidity have been demonstrated to get an important influence with time lags effect on the emergence and transmission of certain rodent-borne infectious diseases $(12,23)$. Lately, people paid more attention to the correlations between meteorological factors, SOI and scrub typhus, and DLNMs, which are widely used to explore the non-linear and lagged relationship, for example, a DLNM result in Taiwan revealed the non-linear and lag relationship between dengue fever and temperature (24). The time lags on scrub typhus may be caused by the life cycle of chigger mites ( $\sim 2-3$ months) (25) and the disease incubation period (average 10 to 12 days) (10).
In our study, we used weekly data to apply DLNMs to explore the relationships between meteorological factors, SOI and scrub typhus cases, which was more accurate comparing to previous studies by monthly data $(19,26)$. What's more, we added the autoregression term to reflect the infectious term in the DLNMs.

Our findings depicted that mean temperature was mainly positively correlated with scrub typhus cases before $29.6^{\circ} \mathrm{C}$, which was consistent with previous studies in India (27) and Korea (26). Biological and ecological evidence can be found to support our findings. Temperature variation can influence the Trombiculidae spawning rates, the abundance and distribution of rodents and the activity of the chiggers. When the temperature increased, the Trombiculidae spawning rates and the abundance of rodents became higher and it promoted the activity of the chiggers (28). Furthermore, warmer condition was more adapted to farm and visit the park (19). Meanwhile, our findings showed that RR was highest when the temperature was $27^{\circ} \mathrm{C}$ with 2 week lag. While the temperature became hotter, people would reduce the willingness to farm or go outside such as a park. The reasons mentioned above supported our results, and the negative impact of atmospheric pressure on scrub typhus was approximately opposite to the effect of mean temperature, which was consistent with the result of a previous study using the negative binomial regression in Guangzhou (29). Unfortunately, the study about the impact of atmospheric pressure on scrub typhus was sparse, a possible reason for this phenomenon is that high atmospheric pressure is not conducive to the survival of mites (30), the biological mechanism should be undertaken in further study.

Temperature range was reported by previous studies to be related to infectious diseases, including influenza (31), hand foot mouth disease (32), and scrub typhus, although the study just pointed out that temperature range was a key determining factor (7). Our study revealed a negative relationship between the WTR and scrub typhus cases. The explanation was as follows. The high WTR usually occur in the winter season in Guangzhou, the Trombiculidae spawning rates and the abundance of rodents were lower and it weakened the activity of the chiggers and reduced the infectious risk of scrub typhus (28). The biological study should be launched in the future. 
Previous studies showed that precipitation had a positive relationship with the occurrence of scrub typhus $(10,19,26)$, which was partially in compliance with our finding. We found that the relative risk increased until the accumulate rainfall reached $40 \mathrm{~mm}$, after which point, the relative risk started to decline. The positive effect on scrub typhus could be related to the increase in precipitation which could increase the growth of vegetation, which directly or indirectly facilitated the survival and reproduction of rodents, leading to higher rodent density (33). As the rainfall increased continuously over $70 \mathrm{~mm}$, it would prohibit people to farm or go outdoor activity, which decreased the contact rate with the chiggers. The above reasons could explain our findings.

Relative humidity was shown to be positively correlated with the incidence of scrub typhus $(10,26)$. Our finding approximately matches with their results, showing a positive correlation with scrub typhus. However, as the relative humidity exceeded the $72 \%$, the relationship was not significant. The biological reasons are as below. When the humidity is low, the adult chiggers stop their spawning. As the relative humidity increased, it offers a moist environment for the growth of mites $(19,29)$. A study in Chile shows that the number or activity of chigger mites decreased when the relative humidity is lower than 50\%, as the relative humidity exceeds $50 \%$, chigger mites can survive and reproduce well (34). However, as the continuous increase of relative humidity over $72 \%$, it was often coincided with rainy day, and the effect of relative humidity may be offset by the effect of precipitation mentioned above. Hence, no statistically significant effect was observed after $72 \%$ relative humidity. This reason could partially explain our findings.

A study in Korea showed that the wind speed was correlated to scrub typhus (26). Our result illustrated that the RR rose sharply as the wind speed increased, however, after the wind speed exceeded $1.6 \mathrm{~m} / \mathrm{s}$, the RR dropper consecutively. The extremely high effect of wind speed showed the RR as 0.88 . There was little research to explain the potential mechanism about the effect of wind velocity on scrub typhus. The study in Korea indicated the wind speed may be correlated to the chigger spawning condition (26). The gale weather is often accompanied by rain, which may influence the activity of people to farm or sit on the grass. However, further studies about the effect of wind speed on the ecology of the chigger lift cycle and infection mechanism should be done in future.

The Sunshine hours have been found in previous studies to have a positive association with scrub typhus cases (29, 35), however our result showed no statistical significance on the overall effect but extreme effect between the sunshine hours and scrub typhus. The RRs of extreme low effects of sunshine hours were 0.81 with 0 -week lag and 1.12 with 10 -week lag. When the duration of sunshine hours is short, it often coincided with precipitation, which reduces the exposure of people to the chiggers. The 2-week lag was consistent with the incubation of scrub typhus. Meanwhile, the precipitation was beneficial for the rodents and chigger to thrive (29). Hence, the RR increased and peaked in lag week 10. It gave us a hint that we should prepare for the control and prevention of scrub typhus when the storm season came.

The Southern Oscillation Index (SOI) reflects global features rather than local meteorological factors (14), which was useful for understanding the long-term trends of tsutsugamushi disease. When we took the larger geographic area climate factors into consideration, it could avoid the collinearity and make the trends more reasonable (36). Previous studies found that the SOI was correlated with some infectious diseases, including dengue fever, and chikungunya $(37,38)$. Our findings reported that the SOI showed an M-shaped curve relationship with scrub typhus cases. Sustained negative values of the SOI below -7 often indicate El Niño episodes, which would cause warmer and more precipitation in Guangzhou. While the sustained positive values over 7 are typical of a La Niña episode, which would cause cooler in autumn and winter in Guangzhou (39). The El Niño and La Niña episodes could cause the variation of temperature and precipitation (40). This finding was consistent with the distribution of Scrub typhus incidence in Guangzhou, Southern China, which was seasonal high from May to October and peaked in June. It was also in accord with our result about the mean temperature and aggregate rainfall, which would affect the density and activity of rodents and chiggers, also influence human behavior. For example, as the positive SOI value increased, the temperature would become cooler and cooler, and our result revealed that the RR values of SOI were below 1 beyond 31.0, and the RR values of mean temperature were lower than 1 below $23.5^{\circ} \mathrm{C}$. However, the internal mechanism of the SOI to either meteorological variable in our study or scrub typhus incidence was complex (41), which need to be further explored as many other factors, including vector, social and human behavior factors, can influence the incidence of scrub typhus.

Some limitations should be mentioned. First of all, we just used the data in Guangzhou, Southern China, which cannot represent other regions. Secondly, the detailed epidemiology investigation about tract event and route of infection for each scrub typhus cases were not available, which affect our interpretation of the effect in the high-risk occupation people. Finally, potential confounding variables could not be excluded in our analyses, for example, host susceptibility, vector factors, population density, and geographic factors. Investigations for these limitations should be launched in further study.

\section{CONCLUSIONS}

In general, meteorological factors and SOI played an important role in scrub typhus occurrence in Guangzhou. Non-linear relationships were observed in almost all the variables in our study. Approximately, mean temperature and relative humidity positively correlated to the incidence of scrub typhus, on the contrary to the atmospheric pressure, and the WTR. Aggregate rainfall and wind velocity showed an inverse- $U$ relationship curve, whereas the SOI appeared the bimodal distribution. These findings provide preliminary, but basic information to better understand the epidemic trends of scrub typhus in Guangzhou, 
southern China. And the result facilitates the development of the early warning system by meteorological factors and SOI surveillance to strengthen the prevention and control of scrub typhus.

\section{DATA AVAILABILITY STATEMENT}

The raw data supporting the conclusions of this article will be made available by the authors, without undue reservation.

\section{ETHICS STATEMENT}

This study got the permission from the ethics committee of the Guangzhou Center for Disease Control and Prevention. Our study did not involve any private and personal information about scrub typhus cases. The data was anonymous.

\section{AUTHOR CONTRIBUTIONS}

ZY: conceptualization and writing-review and editing. JL, YL, $\mathrm{XM}$, and ML: data curation. JL and XM: methodology. ZY and ML: supervision. JL and ZY: validation. JL and YL: visualization.

\section{REFERENCES}

1. Kelly DJ, Fuerst PA, Ching WM, Richards AL. Scrub typhus: the geographic distribution of phenotypic and genotypic variants of Orientia tsutsugamushi. Clin Infect Dis. (2009) 48(Suppl 3):S203-30. doi: 10.1086/596576

2. Park SW, Ha NY, Ryu B, Bang JH, Song H, Kim Y, et al. Urbanization of scrub typhus disease in South Korea. PLoS Negl Trop Dis. (2015) 9:e0003814. doi: 10.1371/journal.pntd.0003814

3. Bonell A, Lubell Y, Newton PN, Crump JA, Paris DH. Estimating the burden of scrub typhus: a systematic review. PLoS Negl Trop Dis. (2017) 11:e0005838. doi: 10.1371/journal.pntd.0005838

4. Wu YC, Qian Q, Magalhaes RJ, Han ZH, Haque U, Weppelmann TA, et al. Rapid increase in scrub typhus incidence in mainland China, 2006-2014. Am J Trop Med Hygiene. (2016) 94:532-6. doi: 10.4269/aitmh.15-0663

5. Sun Y, Wei YH, Yang Y, Ma Y, de Vlas SJ, Yao HW, et al. Rapid increase of scrub typhus incidence in Guangzhou, southern China, 2006-2014. BMC Infect Dis. (2017) 17:13. doi: 10.1186/s12879-016-2153-3

6. Long J, Wei Y, Tao X, He P, Xu J, Wu X, et al. Representative genotyping, recombination and evolutionary dynamics analysis of TSA56 gene segment of Orientia tsutsugamushi. Front Cell Infect Microbiol. (2020) 10:383. doi: $10.3389 /$ fcimb. 2020.00383

7. Yu H, Sun C, Liu W, Li Z, Tan Z, Wang X, et al. Scrub typhus in Jiangsu Province, China: epidemiologic features and spatial risk analysis. BMC Infect Dis. (2018) 18:372. doi: 10.1186/s12879-018-3271-x

8. Du Z, Wang Z, Liu Y, Wang H, Xue F, Liu Y. Ecological niche modeling for predicting the potential risk areas of severe fever with thrombocytopenia syndrome. Int J Infect Dis. (2014) 26:1-8. doi: 10.1016/j.ijid.2014.04.006

9. Walker DH. Scrub typhus - scientific neglect, ever-widening impact. N Engl J Med. (2016) 375:913-5. doi: 10.1056/NEJMp1608499

10. Yang LP, Liu J, Wang XJ, Ma W, Jia CX, Jiang BF. Effects of meteorological factors on scrub typhus in a temperate region of China. Epidemiol Infect. (2014) 142:2217-26. doi: 10.1017/S0950268813003208

11. Vallee J, Thaojaikong T, Moore CE, Phetsouvanh R, Richards AL, Souris M, et al. Contrasting spatial distribution and risk factors for past infection with scrub typhus and murine typhus in Vientiane City, Lao PDR. PLoS Negl Trop Dis. (2010) 4:e909. doi: 10.1371/journal.pntd.0000909

12. Xiang J, Hansen A, Liu Q, Tong MX, Liu X, Sun Y, et al. Impact of meteorological factors on hemorrhagic fever with renal syndrome in
JL, YL, and XM: writing-original-draft. All authors contributed to the article and approved the submitted version.

\section{FUNDING}

This work was supported by the Medical Health Technology Project for Guangzhou (20201A010045, 20201A011062), the Key Project of Medicine Discipline of Guangzhou (No. 2021-2023-11), Science and Technology Planning Project in Guangzhou (201804010093), and Medical Science and Technology Foundation of Guangdong Province (A2021372).

\section{ACKNOWLEDGMENTS}

We appreciate the Guangzhou Meteorological Bureau providing meteorological data for our study.

\section{SUPPLEMENTARY MATERIAL}

The Supplementary Material for this article can be found online at: https://www.frontiersin.org/articles/10.3389/fmed. 2021.667549/full\#supplementary-material
19 cities in China, 2005-2014. Sci Total Environ. (2018) 636:1249-56. doi: 10.1016/j.scitotenv.2018.04.407

13. Semenza JC, Suk JE. Vector-borne diseases and climate change: a European perspective. FEMS Microbiol Lett. (2018) 365:fnx244. doi: $10.1093 / \mathrm{femsle} / \mathrm{fnx} 244$

14. Latif M, Keenlyside NS. El Nino/Southern Oscillation response to global warming. Proc Natl Acad Sci USA. (2009) 106:20578-83. doi: 10.1073/pnas.0710860105

15. Power SB, Kociuba G. The impact of global warming on the Southern Oscillation Index. Clim Dyn. (2011) 37:1745-54. doi: 10.1007/s00382-010-0951-7

16. Xiao H, Tian HY, Cazelles B, Li XJ, Tong SL, Gao LD, et al. Atmospheric moisture variability and transmission of hemorrhagic fever with renal syndrome in Changsha City, Mainland China, 1991-2010. PLoS Negl Trop Dis. (2013) 7:e2260. doi: 10.1371/journal.pntd.0002260

17. Silva-Caso W, Espinoza-Espiritu W, Espejo-Evaristo J, Carrillo-Ng H, Aguilar-Luis MA, Stimmler L, et al. Geographical distribution, evaluation of risk of dengue and its relationship with the El Nino Southern Oscillation in an endemic region of Peru between 2004 and 2015. BMC Res Notes. (2019) 12:498. doi: 10.1186/s13104-019-4537-0

18. Gasparrini A. Distributed lag linear and non-linear models in R: the Package dlnm. J Stat Softw. (2011) 43:1-20. doi: 10.18637/jss.v043.i08

19. Wei Y, Huang Y, Li X, Ma Y, Tao X, Wu X, et al. Climate variability, animal reservoir and transmission of scrub typhus in Southern China. PLoS Negl Trop Dis. (2017) 11:e0005447. doi: 10.1371/journal.pntd.0005447

20. Guo J, Deng M, Lee SS, Wang F, Li Z, Zhai HLP, et al. Delaying precipitation and lightning by air pollution over the Pearl River Delta. Part I_ Observational analyses. J Geophys Res Atmos. (2016) 121:6472-88. doi: 10.1002/2015JD023257

21. Imai C, Armstrong B, Chalabi Z, Mangtani P, Hashizume M. Time series regression model for infectious disease and weather. Environ Res. (2015) 142:319-27. doi: 10.1016/j.envres.2015.06.040

22. Wardrop NA, Kuo CC, Wang HC, Clements AC, Lee PF, Atkinson PM. Bayesian spatial modelling and the significance of agricultural land use to scrub typhus infection in Taiwan. Geospatial Health. (2013) 8:229-39. doi: 10.4081 /gh.2013.69

23. Eisen RJ, Eisen L, Ogden NH, Beard CB. Linkages of weather and climate with Ixodes scapularis and Ixodes pacificus (Acari: Ixodidae), Enzootic 
Transmission of Borrelia burgdorferi, and Lyme Disease in North America. J Med Entomol. (2016) 53:250-61. doi: 10.1093/jme/tjv199

24. Chuang TW, Chaves LF, Chen PJ. Effects of local and regional climatic fluctuations on dengue outbreaks in southern Taiwan. PLoS ONE. (2017) 12:e0178698. doi: 10.1371/journal.pone.0178698

25. Traub R, Wisseman CL. Jr., Ecological considerations in scrub typhus. 2. Vector species. Bull World Health Organ. (1968) 39:219-30.

26. Kwak J, Kim S, Kim G, Singh VP, Hong S, Kim HS. Scrub typhus incidence modeling with meteorological factors in South Korea. Int J Environ Res Public Health. (2015) 12:7254-73. doi: 10.3390/ijerph120707254

27. Dorji K, Phuentshok Y, Zangpo T, Dorjee S, Dorjee C, Jolly P, et al. Clinical and epidemiological patterns of scrub typhus, an emerging disease in Bhutan. Trop Med Infect Dis. (2019) 4: doi: 10.3390/tropicalmed40 20056

28. Van Peenen PF, Lien JC, Santana FJ, See R. Correlation of chigger abundance with temperature at a hyperendemic focus of scrub typhus. J Parasitol. (1976) 62:653-4. doi: 10.2307/3279442

29. Li T, Yang Z, Dong Z, Wang M. Meteorological factors and risk of scrub typhus in Guangzhou, southern China, 2006-2012. BMC Infect Dis. (2014) 14:139. doi: 10.1186/1471-2334-14-139

30. Traub R, Wisseman CL. Jr., Ecological considerations in scrub typhus. 3. Methods of area control. Bull World Health Organ. (1968) 39:231-7.

31. Park JE, Son WS, Ryu Y, Choi SB, Kwon O, Ahn I. Effects of temperature, humidity, and diurnal temperature range on influenza incidence in a temperate region. Influenza Other Respir Viruses. (2019) 14:11-8. doi: 10.1111/irv.12682

32. Hu Y, Liu F, Zhao X, Ma Y, Lan T, Yang F, et al. The modification effect of the diurnal temperature range on the exposure-response relationship between temperature and pediatric hand, foot and mouth disease. Sci Total Environ. (2020) 722:137921. doi: 10.1016/j.scitotenv.2020.1 37921

33. Zhang WY, Wang LY, Liu YX, Yin WW, Hu WB, Magalhaes RJ, et al. Spatiotemporal transmission dynamics of hemorrhagic fever with renal syndrome in China, 2005-2012. PLoS Negl Trop Dis. (2014) 8:e3344. doi: 10.1371/journal.pntd.0003344

34. Abarca K, Weitzel T, Martinez-Valdebenito C, Acosta-Jamett G. Scrub typhus, an emerging infectious disease in Chile. Rev Chilena Infectol. (2018) 35:696-9. doi: 10.4067/S0716-101820180006 00696
35. Tsai PJ, Yeh HC. Scrub typhus islands in the Taiwan area and the association between scrub typhus disease and forest land use and farmer population density: geographically weighted regression. BMC Infect Dis. (2013) 13:191. doi: 10.1186/1471-2334-13-191

36. Oluwole OS. Climate regimes, El Nino-Southern Oscillation, and Meningococcal Meningitis Epidemics. Front Public Health. (2015) 3:187. doi: $10.3389 /$ fpubh.2015.00187

37. Hu W, Clements A, Williams G, Tong S. Dengue fever and El Nino/Southern Oscillation in Queensland, Australia: a time series predictive model. Occup Environ Med. (2010) 67:307-11. doi: 10.1136/oem.2008.044966

38. Huang X, Hu W, Yakob L, Devine GJ, McGraw EA, Jansen CC, et al. El Nino Southern Oscillation, overseas arrivals and imported chikungunya cases in Australia: a time series analysis. PLoS Negl Trop Dis. (2019) 13:e0007376. doi: 10.1371/journal.pntd.0007376

39. Bo AG. Meteorology, Southern Oscillation Index (2019).

40. Zhu D, Xiong K, Xiao H, Gu X. Variation characteristics of rainfall erosivity in Guizhou Province and the correlation with the El Nino Southern Oscillation. Sci Total Environ. (2019) 691:835-47. doi: 10.1016/j.scitotenv.2019.07.150

41. Wang $\mathrm{B}$, Wu R, Fu X. Pacific-East Asian Teleconnection: how Does ENSO Affect East Asian Climate? J Clim. (2000) 13:1517-36. doi: 10.1175/1520-0442(2000)013<1517:PEATHD >2.0.CO;2

Conflict of Interest: The authors declare that the research was conducted in the absence of any commercial or financial relationships that could be construed as a potential conflict of interest.

Publisher's Note: All claims expressed in this article are solely those of the authors and do not necessarily represent those of their affiliated organizations, or those of the publisher, the editors and the reviewers. Any product that may be evaluated in this article, or claim that may be made by its manufacturer, is not guaranteed or endorsed by the publisher.

Copyright $\odot 2021 \mathrm{Lu}, \mathrm{Liu}, \mathrm{Ma}$, Li and Yang. This is an open-access article distributed under the terms of the Creative Commons Attribution License (CC BY). The use, distribution or reproduction in other forums is permitted, provided the original author(s) and the copyright owner(s) are credited and that the original publication in this journal is cited, in accordance with accepted academic practice. No use, distribution or reproduction is permitted which does not comply with these terms. 